\title{
O impacto do edentulismo na qualidade de vida: autoestima e saúde geral do indivíduo
}

\author{
The impact of edentulism on quality of life: self- steem and general health of the individual
}

\section{El impacto del edentulismo en la calidad de vida: autoestima y salud general del individuo}

\author{
Viviane da Silva Izaque ${ }^{1 *}$, Luiz Felipe Gilson de Oliveira Rangel ${ }^{2}$, Athaluama Pires da Silva Inocencio ${ }^{3}$, \\ Carlos Roberto Teixeira Rodrigues ${ }^{4}$ \\ Como citar esse artigo. Izaque, V.S

\section{Resumo}

Rangel, L.F.G.O; Inocêncio, A. P.

S; Rodrigues, C. R. T. O impacto do edentulismo na qualidade de vida autoestima e saúde geral do indivíduo. Revista Pró-UniverSUS. 2021 Jul./Dez. 12 (2): $48-54$

O edentulismo é definido como a perda total dos dentes naturais. Trata-se de um importante problema de saúde pública a nível mundial devido à sua alta prevalência. O objetivo deste estudo foi descrever sobre impacto do edentulismo na saúde oral e geral. Foi realizada uma revisão de literatura, acessando-se estudos nos idiomas português e inglês, publicados entre 2012 e 2019 , indexados na base de dados do Google Acadêmico, acessando-se a pesquisa avançada. Verifica-se que o edentulismo traz consigo uma série de consequências prejudiciais à saúde oral e geral. Entre seus impactos, destacam-se prejuízos funcionais, nutricionais, estéticos, psicológicos e sociais, os quais interferem negativamente na qualidade de vida e autoestima da pessoa. Consistindo também em maior risco de doenças sistêmicas, sobretudo, de origem cardiovascular e metabólica, e, consequentemente, maior risco de mortalidade. Considera-se que há necessidade de desenvolvimento de estratégias de promoção em educação em saúde oral entre as populações de risco, sobretudo na atenção básica de saúde, como forma de reduzir o edentulismo e promover melhorias na qualidade de vida dessas pessoas.

Palavras-chave: Perda de dentes; Boca Edêntula; Saúde Bucal; Saúde Geral.

\begin{abstract}
Edentulism is defined as the total loss of natural teeth. It is a major public health problem worldwide due to its high prevalence. The aim of this study was to describe the impact of edentulism on oral and general health. A literature review was conducted accessing studies in Portuguese and English, published between 2012 and 2019, indexed in the Google Academic database, accessing the advanced search. Edentulism has a number of adverse consequences for oral and general health. Among its impacts are functional, nutritional, aesthetic, psychological and social damage, which negatively interfere with the quality of life and self-esteem of the person. It also consists of a higher risk of systemic diseases, especially of cardiovascular and metabolic origin, and, consequently, a higher risk of mortality. It is considered that there is a need to develop strategies for promoting oral health education among at-risk populations, especially in primary health care, as a way to reduce edentulism and promote improvements in the quality of life of these people.
\end{abstract}

Keywords: Tooth Loss; Mouth Edentulous; Oral Health; General Health.

${ }^{1}$ Acadêmica no Curso de Odontologia, Universidade de Vassouras, Vassouras, RJ, Brasil. E-mail: vivi.enfermeira@hotmail.com ORCID: https://orcid.org/0000-0002-2490-3998 ${ }_{2}^{2}$ Mestre. Professor do Curso de Odontologia, Universidade de Vassouras, Vassouras, RJ, Brasil. E-mail: dr.felipegilsonrangel@gmail.com ORCID: https://orcid.org/0000-0002-73762829

${ }^{3}$ Mestre. Professora do Curso de Odontologia, Universidade de Vassouras, Vassouras, RJ, Brasil. E-mail: athaluamapires@gmail.com ORCID: https://orcid.org/0000-0001-58694357

${ }^{4}$ Doutor. Professor do Curso de Odontologia, Universidade de Vassouras, Vassouras, RJ, Brasil. E-mail: rodriguescrt@gmail.com ORCID: https://orcid.org/0000-0001-6218-1706 


\section{Resumen}

El edentulismo se define como la pérdida total de dientes naturales. Es un importante problema de salud pública a nivel mundial debido a su alta prevalencia. El objetivo de este estudio fue describir el impacto del edentulismo en la salud bucal y general. Se realizó una revisión de la literatura, accediendo a estudios en portugués e inglés, publicados entre 2012 y 2019, indexados en la base de datos de Google Scholar, accediendo a la búsqueda avanzada. Resulta que el edentulismo trae consigo una serie de consecuencias nocivas para la salud bucal y general. Entre sus impactos destacan los daños funcionales, nutricionales, estéticos, psicológicos y sociales, que interfieren negativamente en la calidad de vida y la autoestima de la persona. También consiste en un mayor riesgo de enfermedades sistémicas, especialmente de origen cardiovascular y metabólico, y, en consecuencia, un mayor riesgo de mortalidad. Se considera que es necesario desarrollar estrategias para promover la educación en salud bucal entre las poblaciones en riesgo, especialmente en la atención primaria de salud, como una forma de reducir el edentulismo y promover mejoras en la calidad de vida de estas personas.

Palabras clave: Pérdida de Dientes; Boca Edéntula; Salud Bucal; Salud General.

\section{Introdução}

Uma dentição adequada tem implicações relevantes no bem-estar e na qualidade de vida das pessoas ${ }^{1}$. Por outro lado, a perda dentária tem efeitos deletérios sobre a saúde bucal e geral, provocando danos funcionais, psicossociais e estéticos ${ }^{2}$.

De etiologia multifatorial, o edentulismo é uma condição debilitante que representa o desfecho de um processo contínuo de doenças bucais e sistêmicas. Tratase de um fenômeno complexo, no qual estão envolvidos fatores físicos, biológicos, culturais, econômicos, sociais e comportamentais ${ }^{3}$.

$\mathrm{Na}$ maioria das vezes é resultado de práticas voltadas para extrações dentárias subsequentes a agravos bucais, como a cárie e problemas periodontais ${ }^{4}$. Sendo a cárie dentária a principal causa de edentulismo entre pessoas com menos de 45 anos, enquanto a doença periodontal, o motivo principal entre indivíduos acima dessa faixa etária 5 .

Além disso, a crença de que a perda dentária seja uma consequência natural do envelhecimento, tem contribuído para o negligenciamento dos cuidados bucais e para a gradual substituição dos dentes naturais por próteses dentárias ${ }^{6}$. Situação que poderia ser evitada se houvesse mais orientação, atuação preventiva e cuidados adequados em saúde bucal ${ }^{4}$.

O edentulismo está associado a uma diversidade de efeitos adversos à saúde, além de comprometer a saúde oral, tem sido apontado como fator de risco para várias condições sistêmicas ${ }^{7}$. A ausência dentária implica na dificuldade de mastigação e, consequentemente, para uma ingestão nutricional inadequada, o que pode se tornar um grande fator de risco para o surgimento de diversas comorbidades associadas, como a obesidade, diabetes mellitus e doenças cardiovasculares ${ }^{3,8}$.

Mesmo posteriormente à reabilitação oral, com uso de próteses, o edentulismo tem sido associado a impactos significativos nas atividades funcionais e sociais diárias e bem-estar psicológico ${ }^{7}$. Estudos citam que as próteses totais não são suficientes para restabelecer a função oral, eficácia mastigatória e força da mordida ${ }^{1}$.

Diante dessas premissas, este estudo tem como objetivo descrever, através de uma revisão de literatura, sobre o impacto do edentulismo na saúde oral e geral. Sua elaboração se justifica por considerar que, embora tenha havido no Brasil algumas iniciativas públicas que visem à melhoria da saúde bucal da população, fazendo com que alguns indicadores de saúde bucal, sobretudo para a cárie dentária, tenham melhorado no país, dados epidemiológicos confirmam que o edentulismo ainda se configura como um problema grave entre a população brasileira, com grande impacto sobre a saúde oral e geral, associado com um grande número de pessoas adultas e idosas que ainda dependem de próteses removíveis ${ }^{9}$.

\section{Materiais e Métodos}

Trata-se de uma pesquisa exploratória de caráter descritivo e abordagem qualitativa. Para alcançar o objetivo proposto, utilizou-se uma revisão de literatura, que se constitui em um método bastante útil, pois quando bem desenvolvida sobre um tópico relevante pode gerar um grande impacto sobre a prática profissional ${ }^{10}$.

Foram utilizados estudos em português e inglês indexados na base de dados do Google Acadêmico, com recorte temporal referente ao período de 2012 a 2019, selecionados a partir dos seguintes critérios de inclusão: ser artigo escrito em português e inglês, estar de acordo com o tema de interesse e estar disponível gratuitamente e na íntegra nos bancos de dados.

\section{Resultados e Discussão}

\section{Dados Epidemiológicos do Edentulismo}


O edentulismo é definido como a perda total dos dentes naturais. Trata-se de um importante problema de saúde pública a nível mundial devido à sua alta prevalência e incapacidade associada. Diferentemente de outras condições mórbidas crônicas, que são passíveis de tratamento terapêutico, o edentulismo é uma condição definitiva, representando o estágio final da doença periodontal e da cárie dentária ${ }^{5}$.

A influência de fatores socioeconômicos e sociodemográficos como causas do edentulismo tem sido consenso na literatura. Apesar de se observar a diminuição de casos de edentulismo em países desenvolvidos, existe uma tendência oposta em países de baixa e média renda, onde as taxas continuam crescendo ${ }^{5}$. Esta situação é endossada por outros estudos, ao afirmarem que o edentulismo está intimamente associado a fatores socioeconômicos, sendo mais prevalente entre populações de baixa renda, idosos e com as mulheres apresentando taxas mais altas do que os homens ${ }^{1}$.

Embora também ocorra em faixas etárias mais jovens, sobretudo entre indivíduos socioeconomicamente desfavorecidos, a maioria dos edêntulos é formada por pessoas idosas, que fazem uso de próteses completas em um ou em ambos os maxilares. Situação que provavelmente aumentará à medida que a população envelhece e se vivem mais ${ }^{1,5}$.

Também têm contribuído para a sua alta incidência o nível educacional mais baixo, bem como a falta de capacidade do sistema público de saúde em atender à demanda da população por tratamento odontológico. Para um percentual expressivo de pessoas que necessitam desse tipo de sistema de saúde, o único tratamento viável tem sido a extração dentária ${ }^{1,6}$.

Nos Estados Unidos, estudos apontam a existência de nove milhões de pessoas edêntulas. Estudos mais recentes revelam que a prevalência de edentulismo entre pessoas com idade acima de 60 anos alcançou um percentual de $25 \%$ da população do país nessa faixa etária. No Canadá, em 2010, o percentual de edentulismo entre pessoas adultas era de $6,4 \%$, enquanto entre idosos, na faixa de 60 e 79 anos, o percentual alcançava $21,7 \%{ }^{1}$.

Dados epidemiológicos de 2002-2003, referentes ao Brasil, confirmaram o edentulismo como um pro $\neg$ blema grave no país, associado com alta neces $\neg$ sidade protética em adultos e idosos. Levantamento epidemiológico realizado em 2010, quando comparado com os resultados de 2003, revelaram melhoras em relação à perda dentária em adultos, destacando que a média de dentes perdi $\neg$ dos diminuiu de 13,5 em 2003 para 7,4 em 2010. Nos dois estudos (2003 e 2010), o edentulismo entre as pessoas idosas permaneceu quase que inalterado, com percentual próximo de $54 \%$, abrangendo um contingente de mais de três milhões de idosos com necessidade de prótese total em pelo menos um maxilar'.

Pesquisa mais recente, realizada no ano de 2016 pelo Instituto Brasileiro de Geografia e Estatística (IBGE), verificou que $11 \%$ dos brasileiros são totalmente desdentados, correspondendo a um total de 16 milhões de pessoas. Estimando ainda que até o ano de 2020 o número total de indivíduos na faixa etária dos 50 a 74 anos necessitando de prótese dentária total terá um aumento de cerca de $2 \%$ aproximadamente ${ }^{11}$.

Estudos mostram que existem variações micro e macrorregionais na prevalência do edentulismo, comparação direta entre amostras nacionais são dificultadas devido à interferência de diversos fatores, entre estes, destacam-se a educação, circunstâncias econômicas, estilo de vida, conhecimentos e crenças relacionadas à saúde bucal. Revelando ainda que a taxa de edentulismo tende a variar também entre as diferentes regiões de um país, no Canadá há uma grande variação entre as províncias, devido a fatores associados ao acesso à água potável fluoretada e ao tabagismo ${ }^{1}$.

Estudos apresentam a incidência de edentulismo em diferentes países e regiões, a tabela 1, abaixo, adaptada, mostra essa incidência em alguns países ${ }^{1}$.

Tabela 1. Incidência de edentulismo.

\begin{tabular}{|c|c|c|c|c|}
\hline \multicolumn{5}{|c|}{ PREVALÊNCIA DE EDENTULISMO } \\
\hline País & $\begin{array}{c}\text { Ano da } \\
\text { pesquisa }\end{array}$ & $\begin{array}{c}\text { Tamanho } \\
\text { da amostra }\end{array}$ & $\begin{array}{l}\text { Faixa etária } \\
\quad \text { (anos) }\end{array}$ & $\begin{array}{c}\text { Porcentagem } \\
\text { desdentada }\end{array}$ \\
\hline $\begin{array}{l}\text { Estados } \\
\text { Unidos }\end{array}$ & $\begin{array}{l}2009- \\
2010\end{array}$ & $\begin{array}{c}\text { Cerca de } \\
5.000\end{array}$ & $\begin{array}{c}65-74 \\
\geq 75\end{array}$ & $\begin{array}{l}15 \% \\
22 \%\end{array}$ \\
\hline Canadá & $\begin{array}{l}2007- \\
2009\end{array}$ & 6.000 & $\begin{array}{l}20-59 \\
60-79\end{array}$ & $\begin{array}{c}6 \% \\
22 \%\end{array}$ \\
\hline Brasil & $\begin{array}{l}2002- \\
2003\end{array}$ & 5.349 & 65 a 74 & $54,7 \%$ \\
\hline México & $\begin{array}{l}2002- \\
2003\end{array}$ & 54.638654 & $\begin{array}{l}\geq 18 \\
65-74\end{array}$ & $\begin{array}{c}6,3 \% \\
25,5 \%\end{array}$ \\
\hline Turquia & $\begin{array}{l}2004- \\
2005\end{array}$ & 1.545 & $65-74$ & $48,0 \%$ \\
\hline Suécia & 2002 & 16.416 & $55-84$ & $14 \%$ \\
\hline Hungria & 2004 & 4,606 & $\begin{array}{l}65-74 \\
\geq 75\end{array}$ & $\begin{array}{l}19,8 \% \\
38,7 \%\end{array}$ \\
\hline
\end{tabular}

Fonte: Emami et al. (2013). 
No Brasil, os estados mais industrializados tendem a ter taxas menores de edentulismo, quando comparados com outras partes do país, onde a industrialização é menor $^{1}$. Na realidade, o edentulismo aqui no país se constitui em um indicador da desigualdade social, estando associado às condições socioeconômicas de grande parte da população, representando o legado de um modelo odontológico centrado em procedimentos curativos não conservadores, que resultou em uma realidade de muitas exodontias e alta demanda por serviços protéticos ${ }^{12}$

\section{Impacto do Edentulismo na Saúde Oral e Geral}

A Organização Mundial da Saúde (OMS) define saúde bucal como "um estado livre de dores crônicas na boca e na face, câncer de boca e garganta, infecções e feridas orais, doenças periodontal (gengiva), cárie dentária, perda dentária e outras doenças e distúrbios que limitam a capacidade de um indivíduo morder, mastigar, falar e bem-estar psicossocial"13.

Um determinante significativo da função oral e do estado de saúde bucal é o número de dentes que uma pessoa possui. A perda dentária é uma das principais consequências das más condições de saúde bucal, refletindo um efeito cumulativo de doenças bucais ao longo da vida associada à opção pela extração dentária como forma de tratamento odontológico ${ }^{2}$.

Estomatite, inflamação da mucosa palatina, quelite angular, infecção por Cândida albicans e úlceras traumáticas são condições que têm sido associadas ao uso de próteses ${ }^{1,7}$. Existindo aproximadamente três vezes mais chances de usuários de prótese total apresentar hiperplasia, estomatite e quelite angular. Embora a maioria dessas condições seja benigna, algumas podem se tornar malignas, especialmente se houver redução das funções protetoras da mucosa oral ${ }^{1}$.

Estudo realizado em Atenas (Grécia), em que foram analisados, retrospectivamente, 873 prontuários de pacientes geriátricos que usavam próteses dentárias, revelou que o tempo de edentulismo, independentemente da idade, foi associado a um aumento acentuado na prevalência de disfunção da articulação temporomandibular. Enquanto o aumento da idade foi associado a uma tendência de maior prevalência de estomatite ${ }^{7}$.

Existem também relatos de que o edentulismo possa induzir uma discinesia oral, chamada de movimentos orofaciais anormais, sem propósito e involuntários. Embora ainda não estejam bem claros os mecanismos que possam provocar este distúrbio em indivíduos desdentados, sugere-se que esteja associado ao uso de próteses inadequadas e instáveis, desconforto oral e falta de contatos sensoriais. Como também está associado à discinesia tardia, um distúrbio neurológico caracterizado por movimentos involuntários da mandíbula e da face ${ }^{1}$.

A redução da crista alveolar residual é considerada uma das sequelas orais mais importantes do edentulismo ${ }^{7}$. A perda óssea é um processo progressivo após a perda dentária, afetando a mandíbula quatro vezes mais que a maxila. $\mathrm{O}$ edentulismo tem implicação significativa na reabsorção residual da crista, o que leva a uma redução na altura do osso alveolar e no tamanho da área da prótese, causando grande instabilidade da mesma e, consequentemente, ocasionando dificuldade de adaptação, função mastigatória deficiente e dor ${ }^{1,14}$.

A estética da face fica comprometida, pois a reabsorção óssea alveolar afeta tanto as estruturas internas quanto as externas do rosto ${ }^{14}$. Embora essas alterações degenerativas anatômicas possam variar entre os indivíduos, a redução na altura e largura do osso alveolar tem efeito sobre a aparência do rosto, levando a alterações substanciais no perfil dos tecidos moles, como protrusão do lábio, mandíbula e queixo. Uma combinação de fatores contribui para isso, tais como idade, sexo, duração do edentulismo, hábitos funcionais e saúde geral ${ }^{1}$.

A literatura é unânime em afirmar que a perda total dos dentes representa além de tudo, um obstáculo à mastigação e à alimentação saudável. Estudo realizado mostrou que grande parte dos indivíduos tem dificuldades para se alimentar após o edendulismo. ${ }^{15}$

Mesmo aqueles que fazem uso de próteses dentárias, a eficiência mastigatória é significativamente reduzida, os quais têm apenas um quinto a um quarto da força de mordida e da força mastigatória, quando comparados a indivíduos com dentição natural completa. Para poder cortar os alimentos na metade do seu tamanho original, usuários de dentaduras necessitam realizar sete vezes mais movimentos de mastigação do que aqueles com dentições naturais ${ }^{1,11}$.

Além disso, em indivíduos edêntulos a espessura do músculo masseter tende a diminuir, isso pode explicar em parte o motivo dos usuários de próteses totais terem dificuldades em mastigar alimentos mais duros. Essas deficiências influenciam significativamente no desejo de morder, mastigar e engolir, gerando impacto negativo na dieta e na seleção de alimentos, e, consequentemente, no estado nutricional desses indivíduos, que tendem a preferir alimentos macios e facilmente mastigáveis, que geralmente são pobres em fibras e com baixa densidade nutricional ${ }^{1,11}$

Estudos mostram que a ingestão pobre em frutas, vegetais e fibras e maior ingestão de alimentos ricos em gorduras, colesterol e carboidratos implicam em aumento nos níveis de colesterol, de triglicerídeos e de maior incidência de obesidade, aumentando os riscos de doenças cardiovasculares, diabetes mellitus não insulinodependente e distúrbios gastrointestinais ${ }^{1,16}$. 
Resultado de estudo realizado em Londrina-PR, com 489 indivíduos de ambos os gêneros, com idade acima de 60 anos, fisicamente independentes, mostrou que o edentulismo pode ser considerado um dos fatores associados ao risco de complicações metabólicas que levam a enfermidades cardiovasculares entre os idosos $^{16}$.

Estudo realizado com 108 idosos usuários da atenção básica de um município do Rio Grande do Norte, com edentulismo parcial e total revelou que a falta de dentes tem impacto negativo na qualidade de vida dos idosos. Os impactos na saúde sistêmica foram observados com a prevalência de doenças crônicas e elevado Índice de Massa Corporal (IMC) ${ }^{12}$.

Dietas abaixo do ideal recomendado, também podem levar ao comprometimento do estado nutricional. Estudo mostrou que 39\% dos idosos edêntulos foram impedidos de comer os alimentos que realmente gostariam de comer, $29 \%$ relataram ter menos prazer em comer e $14 \%$ evitavam comer junto a outras pessoas ${ }^{1}$.

Existem relatos de que há uma associação entre edentulismo e respiração desordenada do sono, incluindo apneia obstrutiva do sono ${ }^{1}$. Como também implicações significativas na saúde mental, como baixa autoestima, depressão e baixa autoavaliação da saúde entre pessoas em faixas etárias mais jovens ${ }^{5}$. Revelando ainda que $70 \%$ das pessoas têm sua autoconfiança e autoimagem afetadas pela perda dentária ${ }^{15}$.

O edentulismo pode levar a pessoa a evitar participar de atividades sociais porque sente vergonha de falar, sorrir ou comer na frente de outras pessoas, induzindo ao isolamento e declínio do bem-estar psicossocial. Algumas pessoas conseguem superar as limitações das próteses, mas outras são incapazes de fazê-lo ${ }^{1}$.

Estudo realizado com a finalidade de compreender as experiências da perda dentária, revelou que entre idosos sem reabilitação pro $\rightarrow$ tética, a ausência total de dentes está relacionada à incapacidade masti $\neg$ gatória, à estética, à dor e ao convívio social prejudicado, com grande impacto na relação desses indivíduos com o mundo em que vivem? .

Foi observado que o edentulismo entre pessoas idosas, diferente dos mais jovens, está mais associado com doenças crônicas não transmissíveis, como o diabetes, e, junto com estas, compartilham os mesmos fatores de risco, como por exemplos, baixa escolaridade, tabagismo, ingestão de bebidas alcoólicas, entre outros. Sugerindo que esforços para limitar a exposição a estes fatores de risco, também podem contribuir para a redução do edentulismo entre pessoas nessas faixas etárias ${ }^{5}$.

Um estudo apontou impacto negativo da perda dentária na qualidade de vida de indivíduos adultos jovens, na faixa etária de 18 a 39 anos. Revelando que o edentulismo entre os pacientes estava diretamente relacionado com problemas fonéticos, mastigatórios, com maior probabilidade de adquirir doenças sistêmicas, problemas estéticos e de ordem psicossocial, consequentemente, contribuindo para redução da qualidade de vida desses indivíduos ${ }^{8}$.

Qualidade de vida é a denominação de um termo que pode indicar a presença de saúde física e psíquica, acesso a uma boa educação, boa alimentação, a um serviço de saúde adequado como também a inserção no meio social. Dessa forma, representa um fator positivo para que o indivíduo viva bem e consiga realizar todas as suas tarefas com êxito ${ }^{17}$.

O edentulismo é resultante de doenças e condições que afetam o complexo bucal, classificandose desta forma as perdas dentais como resultantes de fatores biológicos ${ }^{18,1}$. Entretanto estas perdas podem ser também uma consequência de fatores não relacionados a patologias, como por exemplo, a falta de acesso aos serviços de saúde, sendo então relacionadas a fatores não biológicos. Portanto, as razões que explicam as perdas dentais derivam de uma relação multifatorial que compreende aspectos fisiológicos, individuais, culturais e socioeconômicos ${ }^{19}$.

Estudos mostram que apesar da crescente diminuição das taxas de edentulismo, ocorrida pelo melhor acesso à informação e aos serviços de saúde; dentre outros fatores, esta condição ainda continua expressiva sendo um dos grandes problemas de saúde oral em todo o mundo ${ }^{6,1}$. Há ainda um maior número de casos de edentulismo em regiões de baixa renda, idosos, e indivíduos do sexo feminino, demostrando assim a associação entre o edentulismo e fatores socioeconômicos ${ }^{1}$.

No Brasil, de uma maneira geral, a saúde bucal dos idosos é precária e caracterizada por perdas dentárias, infecções e higiene bucal inadequada. Em relação à associação que se fazem sobre o edentulismo como um processo natural do envelhecimento, adverte que pode ser evitado com orientação, ação preventiva $\mathrm{e}$ cuidados adequados de saúde bucal ${ }^{4}$.

Vários estudos apontam uma forte correlação entre o edentulismo e fatores socioeconômicos, em que, por exemplo, o acesso dificultado aos cuidados em saúde e a informação, tornou os indivíduos propensos a perder elementos dentais ${ }^{20,21}$.

Apesar dos avanços obtidos em relação à saúde bucal da população, ainda é notório a persistência de desigualdades sociais e regionais, o que leva a sugerir que, ao lado de medidas universais, deva se priorizar o cuidado entre as populações mais vulneráveis ${ }^{2}$.

$\mathrm{O}$ acesso universal aos serviços médicos e odontológicos da rede pública de saúde nem sempre é possível, como também nem sempre há a garantia de tratamentos e assistência recomendada. Diante desse cenário, os impactos na qualidade de vida dos idosos são inevitáveis. Isso requer que se dê maior atenção 
na elaboração de políticas públicas voltadas para essa camada da sociedade ${ }^{4}$.

Estudos mostram que as condições econômicas e sociais têm maior peso em relação ao edentulismo do que as características raciais dos indivíduos. Evidencia-se também, que a pobreza, baixa escolaridade, localidades com menores coberturas de fluoretação de águas e serviços odontológicos e higiene oral inadequada são fatores que contribuem para o aumento da prevalência e extensão da cárie dentária e, consequentemente, das perdas dela resultantes ${ }^{2}$.

Assim, a perda total de dentes (edentulismo), ainda é aceita pela sociedade como algo normal e natural com o avanço da idade, e não como reflexo da falta de políticas públicas no campo da saúde bucal, destinadas principalmente à população adulta, para que mantenha seus dentes até idades mais avançadas ${ }^{22}$.

Além das desigualdades socioeconômicas, o edentulismo também é reflexo da precária educação em saúde bucal, atitudes do dentista, políticas de assistência bucal, como também a dificuldade de acesso ao tratamento odontológico ${ }^{5}$.

A necessidade de prótese total diminui à medida que os níveis educacionais aumentam, justificando que as pessoas mais instruídas buscam por tratamento com maior antecedência do que aqueles menos instruídos. Assim como o nível educacional, a renda familiar também influencia na qualidade de vida dos indivíduos ${ }^{21}$.

$O$ ser humano vive em busca de satisfação constante, e tudo o que ele quer é ser feliz. O convívio diário com outros seres humanos faz com que interaja constantemente e que tenha que administrar suas próprias necessidades de ser, estar, ter, sentir, conhecer, fazer ${ }^{23}$.

Por meio do processo de melhoria da qualidade de vida, busca-se um estado permanente de equilíbrio entre a saúde física, o domínio, vivência das emoções e a elevação da consciência através de valores e crenças. A partir desse equilíbrio pessoal e interior as relações interpessoais agregam qualidade e busca-se uma harmonia com o meio ambiente ${ }^{24}$.

Como consequências do edentulismo, se tem a limitação funcional, incapacidade física e comprometimento social e psicológico. O que significa que o impacto do edentulismo na saúde geral do indivíduo deve ser avaliado pela análise das principais dimensões da saúde, como por exemplos, pelos sintomas físicos e capacidade funcional, atividades sociais e percepção do bem-estar. Diante das inúmeras consequências deletérias geradas pelo edentulismo na saúde bucal e geral é preciso que os prestadores de serviços em saúde bucal trabalhem em prol de evitar a perda dentária por meio da educação e promoção da saúde bucal, atendimento odontológico adequado, de forma a garantir a existência de uma dentição fisiológica ${ }^{1}$.

Condições sistêmicas crônicas como diabetes mellitus tipo 2, hipertensão, obesidade e problemas de saúde mental, entre outros podes ser atribuída individual e coletivamente à perda precoce de dentes. Fatores sociais insatisfatórios em conjunto com problemas de saúde sistêmica possivelmente exercem um papel cada vez mais importante na etiologia da doença oral continuada e, por fim, no edentulismo precoce ${ }^{3}$.

Além disso, diante da existência de um serviço odontológico restaurador necessário à reabilitação do edentulismo com próteses funcionais e estéticas ser subótimo e inacessível à grande maioria das pessoas, devido a limitações de recursos ou dificuldades para atender os diferentes casos existentes, o edentulismo também pode ter um efeito negativo na saúde mental de indivíduos em situações econômicas desfavoráveis ${ }^{4,5}$.

Outro fator importante o qual sofre influência direta do edentulismo é a estética, que pode resultar em diminuição da autoestima dando origem a alterações psicológicas. Na grande maioria dos casos, os pacientes edêntulos sentem-se insatisfeitos com sua imagem e acaba por se ausentar dos círculos sociais, o que também representa fator de impacto negativo na qualidade de vida $^{18}$.

\section{Considerações Finais}

Este estudo revelou que o edentulismo é um sério problema de saúde pública a nível mundial, com alta prevalência entre as pessoas idosas. Essa é uma situação que pode se tornar ainda mais séria devido às mudanças demográficas que vem ocorrendo no mundo. $\mathrm{O}$ aumento da população idosa e, consequente aumento de doenças crônicas que acompanham o envelhecimento podem indiretamente ser agravadas pelo edentulismo.

Pode-se inferir que o edentulismo acarreta uma série de agravos à saúde oral e geral dos indivíduos. Com destaque para a função mastigatória prejudicada, a qual traz consequências deletérias à saúde geral do indivíduo, devido à ingestão de uma dieta inadequada e consequentes problemas nutricionais. Foi constatado ainda que o edentulismo tem impacto negativo na saúde mental, nas relações sociais, na qualidade de vida e na autoestima das pessoas.

Considera-se que há necessidade de desenvolvimento de estratégias de promoção em educação em saúde oral entre as populações de risco, sobretudo na atenção primária de saúde, como forma de reduzir o edentulismo e promover melhorias na qualidade de vida das pessoas

\section{Referências}

1. Emami E, Souza RF, Kabawat M, Feine JS. The impact of edentulism on oral and general health. Int J Dent. 2013:498305. 
2. Peres MA, Barbato PR, Reis SCGB, Freitas CHSM, Antunes JLF. Perdas dentárias no Brasil: análise da pesquisa nacional de saúde bucal 2010. Rev Saúde Públ. 2013;47Supl 3:S78-89.

3. Latif TM, Vieira AR. Risk factors and comorbidities associated with complete edentulism in individuals younger than fifty years of age. J Dent Oral Health. 2017; 4:1-6.

4. Oliveira FTS. O impacto do edentulismo na qualidade de vida de idosos. [Monografia] - Campos Gerais: Universidade Federal de Minas Gerais;2013.

5. Tyrovolas S, Koyanagi A, Panagiotakos DB, Haro JM, Kassebaum NJ, Chrepa V, et al. Population prevalence of edentulism and its association with depression and self-rated health. Sci Rep. 2016; 6:1-9.

6. Cardoso M, Balducci I, Telles DM, Lourenço EJV, Nogueira Júnior L. Edentulismo no Brasil: tendências, projeções e expectativas até 2040. Ciênc. Saúde Colet. Abr. 2016; 21(4):1239-1246.

7. Divaris K, Ntounis A, Marinis A, Polyzois G, Polychronopoulou A. Loss of natural dentition: multi-level effects among a geriatric population. Gerodontology. 2012;29(2):192-199.

8. Carvalho LF, Melo JRO, Ramos JG, Lima RA, Carvalho FAA. $\mathrm{O}$ impacto do edentulismo na qualidade de vida de pacientes edentulos. RvAcBO. 2018;8(1):40-48.

9. Bitencourt FV, Corrêa HW, Toassi RFC. Experiências de perda dentária em usuários adultos e idosos da Atenção Primária à Saúde. Ciênc. SaúdeColet. 2019Jan;24(1):169-180.

10. Otrenti E. Avaliação de processos educativos formais para profissionais da área de saúde: revisão integrativa de literatura. [Dissertação] - São Paulo: Escola de Enfermagem da Universidade de São Paulo,2011.

11. Batista VL. Avaliação da eficiência mastigatória em pacientes portadores de prótese total bimaxilar. [TCC] - Londrina: Universidade Estadual de Londrina, 2018

12. Dantas LRO. Impacto do Edentulismo na qualidade de vida de idosos usuários da atenção básica. J Dent Pub H. 2019; 10(1):1-6.

13. World Health Organization (WHO). Oral health. [Online] Avaliable fron Internet: $<$ https://www.afro.who.int/health-topics/oral-health $>$. [cited 14-4-2019].

14. Machado RMM. Reabsorção óssea mandibular e sua relação com parâmetros objetivos e subjetivos de função mastigatória no desdentado total. [Dissertação] - Pelotas: Universidade Federal de Pelotas, 2014.

15. Probst LF, Ambrosano GMB, Cortellazzi KL, Guerra LM, RibeiroDasilva MC, Tomar SL et al. Fatores associados aos sentimentos decorrentes da perda dentária total e às expectativas de reposição protética em adultos e idosos. Cad. Saúde Colet., 2016, Rio de Janeiro, 24(3):347-354.

16. Peruchi CTR. Relação entre a condição de saúde bucal e alguns marcadores de risco para doenças cardiovasculares em idosos independentes. [Dissertação] - Maringá: Universidade Estadual de Maringá.

17. Cunha M, Santos E, Costa A, Pereira M, Varanda R, Loreiro S. Saúde oral, literacia e qualidade de vida em idosos - Revisão sistemática da literatura. Revista de enfermagem referência, 2014;1(4):125-134.

18. Campos ACV, Vargas AMD, Ferreira EF. Satisfação com saúde bucal de idosos brasileiros: um estudo de gênero com modelo hierárquico. Cadernos de Saúde Pública 2014;4(30):757-773.

19. Silva EA, Batista MJ, Sousa MLR. Impacto da saúde bucal na qualidade de vida de adultos de diferentes níveis socioeconômicos. Revista Ciências Médicas 2016;1(25):11-21.

20. Marchi RJD, Leal AF, Padilha DM, Brondani MA. Vulnerability and the psychosocial aspects of tooth loss in old age: A southern Brazilian study. J Cross cult gerontol 2012;27(2):239-258.

21. Hamdan EA, Fahmy MM. Socioeconomic factors and complete edentulism for female patients at King Saud University, Riyadh, Saudi Arabia. Tanta Dental Journa 2014;11(2):169-173.

22. Pucca Jr. GA. A saúde bucal do idoso? Aspectos demográficos e epidemiológicos. Medcenter 2000. [acessado 2005 Set 15]. Disponível em:

http://www.odontologia.com.br/

23. Patrício ZM, Casagrande JL, Araújo MF. (org) Qualidade de vida do trabalhador - uma abordagem qualitativa do ser humano através de novos paradigmas. Florianópolis: do autor, 1999.

24. Lipp M. (org.) O stres está dentro de você. São Paulo: Contexto, 1999 